\title{
LONG-TIME DYNAMICS OF A COUPLED CAHN-HILLIARD-BOUSSINESQ SYSTEM*
}

\author{
$\mathrm{KUN} \mathrm{ZHAO}^{\dagger}$
}

\begin{abstract}
We study large-time asymptotic behavior of classical solutions to an initial-boundary value problem (IBVP) for a coupled Cahn-Hilliard-Boussinesq system on a bounded domain. Sufficient conditions are established under which classical solutions converge exponentially to constant states as time goes to infinity due to diffusion and boundary effects.
\end{abstract}

Key words. Cahn-Hilliard-Boussinesq system, asymptotic behavior.

AMS subject classifications. 35Q35, 35B40.

\section{Introduction}

As one of the fundamental modeling equations, the Cahn-Hilliard equation $([4,5])$ plays an important role in the mathematical study of multi-phase flows, and has been studied intensively in the literature both analytically and numerically (see e.g. $[1,2,6,10,11,17,18,20,21,22,23,24])$. The couplings of the Cahn-Hilliard equation with other basic modeling equations have been proposed in various situations to study complicated phenomena in fluid mechanics involving phase transition. For example, the coupled Cahn-Hilliard-Navier-Stokes (CHNS) system and its variations, which describe the motion of an incompressible two-phase flow under shear through an order parameter formulation, have been used in order to understand the phenomena of phase transition in incompressible fluid flows (c.f. [12, 16, 19]). Recently, a closely related model to the CHNS system has been developed in $[3,8,9,13,14]$ to understand the spinodal decomposition of binary fluid in a Hele-Shaw cell, tumor growth, cell sorting, and two phase flows in porous media, which is referred to as the Cahn-Hilliard-HeleShaw (CHHS) system. In this paper, we consider the following system of equations:

$$
\left\{\begin{array}{l}
\phi_{t}+U \cdot \nabla \phi=\nabla \cdot(\beta(\phi) \nabla \mu), \quad \mathbf{x} \in \mathbb{R}^{n}, t>0 \\
\mu=-\alpha \Delta \phi+F^{\prime}(\phi), \\
U_{t}+U \cdot \nabla U+\nabla P=\mu \nabla \phi+\theta \mathbf{e}_{n} \\
\theta_{t}+U \cdot \nabla \theta=\nabla \cdot(\kappa(\phi) \nabla \theta) \\
\nabla \cdot U=0
\end{array}\right.
$$

which is obtained by coupling the Cahn-Hilliard equation to the inviscid heatconductive Boussinesq equations. It describes the motion of an incompressible inviscid two-phase flow subject to convective heat transfer under the influence of gravitational force through order parameter formulation. Here, $U=\left(u_{1}, \ldots, u_{n}\right), P$, and $\theta$ denote the velocity, pressure, and temperature respectively; $\phi \in[-1,1]$ is the order parameter and $\mu$ is a chemical potential derived from a coarse-grained study of the free energy of the fluid (c.f. [15]); the functions $\beta(\phi)>0$ and $\kappa(\phi)>0$ model diffusion and heat conduction, respectively, whose dependence on $\phi$ will be specified below; the constant $\alpha>0$ is a diffuse interface parameter modeling the thickness of the interface, and $\mathbf{e}_{n}$ is the $n$-th unit vector in $\mathbb{R}^{n}$. The function $F(\cdot)$ appearing in the second equation of (1.1)

\footnotetext{
*Received: March 7, 2011; accepted (in revised version): August 19, 2011. Communicated by John Lowengrub.

${ }^{\dagger}$ Department of Mathematics, University of Iowa, Iowa City, IA 52242, USA (kunzhao@uiowa.edu).
} 
normally has a physical-relevant, double-well structure, each of them representing the two phases of the fluid. A typical example of $F(\cdot)$ takes the form: $F(z)=\frac{1}{4}\left(z^{2}-1\right)^{2}$ (see e.g. $[7,15])$. In this paper, we shall consider a general scenario by imposing appropriate growth conditions on $F$.

In this paper, we consider system (1.1) on a bounded domain in $\mathbb{R}^{n}$. The system is supplemented by the following initial and boundary conditions:

$$
\left\{\begin{array}{l}
(\phi, \mu, U, \theta)(\mathbf{x}, 0)=\left(\phi_{0}, \mu_{0}, U_{0}, \theta_{0}\right)(\mathbf{x}), \quad \mathbf{x} \in \Omega \\
\left.\nabla \phi \cdot \mathbf{n}\right|_{\partial \Omega}=0,\left.\nabla \mu \cdot \mathbf{n}\right|_{\partial \Omega}=0,\left.U \cdot \mathbf{n}\right|_{\partial \Omega}=0,\left.\theta\right|_{\partial \Omega}=\bar{\theta}, \quad t \geq 0
\end{array}\right.
$$

where $\Omega \subset \mathbb{R}^{n}$ is a bounded domain with smooth boundary $\partial \Omega, \mathbf{n}$ is the unit outward normal to $\partial \Omega$ and $\bar{\theta}$ is a constant.

The initial-boundary value problem (1.1)-(1.2) was first studied in [25], where the global existence and uniqueness of classical solutions are established, for large initial data with finite energy in 2D. However, the large time asymptotic behavior of the solutions was not investigated due to the lack of uniform-in-time estimates of the solutions. Suggested by the conservation of total mass and the boundary conditions, it is expected that the global attractor of $\phi$ should be $\bar{\phi}=\frac{1}{|\Omega|} \int_{\Omega} \phi_{0}(\mathbf{x}) d \mathbf{x}$, which is its average over the domain, and the global attractor of $\theta$ should be $\bar{\theta}$, due to diffusion and boundary effects. The purpose of this paper is to give rigorous justification of this conjecture for the $2 \mathrm{D}$ case, based on new findings of the structure of the system and to provide sufficient conditions guaranteeing the decay of the solution.

First, we will show that when the diffuse interface parameter $\alpha$ passes a threshold value, which is determined by the function $F$ and the size of the domain $\Omega, \phi$ and $\theta$ will converge to $\bar{\phi}$ and $\bar{\theta}$ exponentially in time respectively, regardless of the magnitude of the initial perturbation. This rigorously justifies the fact that making the thickness of the diffuse interface (modeled by $\alpha$ ) large in the coupled Cahn-Hilliard type equations leads to constant phase states (e.g. [7]). On the other hand, although it is well-known that constant solutions of the classical Cahn-Hilliard equation are locally asymptotically stable (metastable) when they are outside the chemical spinodal (c.f. [2]), it is highly non-trivial that the same fact holds true if the Cahn-Hilliard equation is coupled with a supplementary transport term $U \cdot \nabla \phi$, and the governing equation of the velocity is of hyperbolic type and is driven by the chemical potential and gravitational force. Second, after switching to an other method, we will show the exponential decay of the solution for small initial perturbations around the constant equilibrium state without the assumption on the diffuse interface parameter $\alpha$. We remark that the former result holds for large initial perturbations around the constant state, which is one of the remarkable advantages of our results. We will also see in Section 3 that the proof of the small perturbation case is more delicate than in the large perturbation case, which is another novelty of this paper.

We briefly explain the ideas used in the proofs. For the large perturbation case, we will require that the diffuse interface parameter $\alpha$ satisfies $\alpha-F_{3} c_{0}>0$, where $F_{3}>0$ is a constant such that $F^{\prime \prime}(\cdot) \geq-F_{3}$ and $c_{0}$ is the constant in Poincaré inequality over the domain $\Omega$, which can be computed explicitly for given $F$ and $\Omega$. The condition is crucial in our analysis due to the fact that it produces a positive constant multiple of $\|\phi-\bar{\phi}\|_{H^{2}}^{2}$, which is one of the major dissipative terms controlling the exponential decay of $\phi$. The condition will trigger a chain reaction leading the energy estimate performed in [25] to a whole new scenario. For the small perturbation case, the proof is in the spirit of [3], where the metastability of constant solutions of the Cahn-HilliardNavier-Stokes equations was established. However, due to the vanishing of viscosity 
in the momentum equation and its further coupling with the temperature fluctuation, the analysis of (1.1) requires more deliberation. The idea is to first assume $F^{\prime \prime}(\cdot) \geq 0$ on a small neighborhood $I_{\bar{\phi}}=[\bar{\phi}-\delta, \bar{\phi}+\delta]$ and then solve a modified problem with $F$ replaced by an auxiliary function $F_{\bar{\phi}}$ whose second order derivative is non-negative on $\mathbb{R}$ and coincides with $F^{\prime \prime}(\cdot)$ on $I_{\bar{\phi}}$. Then, under the smallness assumption on the initial perturbations, it can be shown that the solution to the modified problem is indeed the solution to the original problem and converges exponentially to the constant state as time goes to infinity.

Throughout this paper, $\|\cdot\|_{L^{p}},\|\cdot\|_{L^{\infty}}$, and $\|\cdot\|_{W^{s, p}}$ denote the norms of the usual Lebesgue measurable function spaces $L^{p}(1 \leq p<\infty), L^{\infty}$, and the usual Sobolev space $W^{s, p}$ respectively. For $p=2$, we denote the norm $\|\cdot\|_{L^{2}}$ by $\|\cdot\|$ and $\|\cdot\|_{W^{s, 2}}$ by $\|\cdot\|_{H^{s}}$ respectively. The function spaces under consideration are $C\left([0, T] ; H^{r}(\Omega)\right)$ and $L^{2}\left([0, T] ; H^{s}(\Omega)\right)$, equipped with norms $\sup _{0 \leq t \leq T}\|\Psi(\cdot, t)\|_{H^{r}}$ and $\left(\int_{0}^{T}\|\Psi(\cdot, \tau)\|_{H^{s}}^{2} d \tau\right)^{1 / 2}$, where $r, s$ are positive integers. Unless specified, $c$ will denote a generic constant which is independent of the unknown functions and $t$, but may depend on the system parameters and initial data. The value of $c$ may vary line by line according to the context.

For the sake of completeness, we first state the global existence result regarding classical solutions to (1.1)-(1.2), based on which the asymptotic analysis will be performed leading to the main results of this paper.

TheOREM 1.1 (Global existence [25]). Let $\Omega \subset \mathbb{R}^{2}$ be a bounded domain with smooth boundary. Suppose that $F(\cdot)$ satisfies the following conditions:

- $F(\cdot)$ is of $C^{6}$ class and $F(\cdot) \geq 0$;

- There exist constants $F_{1}, F_{2}>0$ such that $\left|F^{(n)}(\phi)\right| \leq F_{1}|\phi|^{p-n}+F_{2}, \quad n=$ $1, \ldots, 6, \forall 6 \leq p<\infty$ and $\phi \in \mathbb{R}$;

- There exists a constant $F_{3} \geq 0$ such that $F^{\prime \prime} \geq-F_{3}$.

Moreover, suppose that there exist positive constants $\beta_{i}, \kappa_{i}(i=0,1,2)$ such that $\beta_{0} \leq \beta(\phi) \leq \beta_{1},\left|\beta^{\prime}(\phi)\right| \leq \beta_{2}, \kappa_{0} \leq \kappa(\phi) \leq \kappa_{1}$, and $\left|\kappa^{\prime}(\phi)\right| \leq \kappa_{2}$ for any $\phi \in \mathbb{R}$. If $\phi_{0}(\mathbf{x}) \in$ $H^{5}(\Omega), \mu_{0}(\mathbf{x}) \in H^{3}(\Omega)$, and $\left(\theta_{0}(\mathbf{x}), U_{0}(\mathbf{x})\right) \in H^{3}(\Omega)$ are compatible with the boundary conditions, then there exists a unique solution $(\phi, \mu, \theta, U)$ to (1.1)-(1.2) globally in time such that $\phi \in C\left([0, T] ; H^{5}(\Omega)\right) \cap L^{2}\left([0, T] ; H^{7}(\Omega)\right), \mu \in C\left([0, T] ; H^{3}(\Omega)\right) \cap$ $L^{2}\left([0, T] ; H^{5}(\Omega)\right), U \in C\left([0, T] ; H^{3}(\Omega)\right)$, and $\theta \in C\left([0, T] ; H^{3}(\Omega)\right) \cap L^{2}\left([0, T] ; H^{4}(\Omega)\right)$ for any $0<T<\infty$.

The following two theorems are the main results of this paper concerning the large time asymptotic behavior of classical solutions to (1.1)-(1.2). Regarding the large initial perturbation case, we have

TheOREM 1.2. Let the conditions in Theorem 1.0 be in force, and suppose that $\beta(\phi) \equiv$ const. and the constant $\alpha_{1}=\alpha-F_{3} c_{0}>0$, where $c_{0}$ is the constant in the Poincaré inequality on the domain $\Omega$. Then the solution satisfies

$$
\|\phi(\cdot, t)-\bar{\phi}\|_{H^{2}}+\|\theta(\cdot, t)-\bar{\theta}\|_{H^{1}} \leq \gamma_{1} e^{-\eta_{1} t}, \quad \forall t \geq 0,
$$

for some constants $\gamma_{1}, \eta_{1}>0$ which are independent of $t$, where $\bar{\phi}$ is the average of $\phi_{0}$ over $\Omega$.

When the initial perturbation is small, we have

TheOREM 1.3. Let the conditions in Theorem 1.0 be in force, and suppose that for some fixed $0<\eta<\bar{\phi}$ it holds that $F^{\prime \prime}(\cdot) \geq 0$ on $[\bar{\phi}-\eta, \bar{\phi}+\eta]$, and there exists a small 
constant $\varepsilon>0$ such that $\left\|U_{0}\right\|^{2}+\left\|\phi_{0}-\bar{\phi}\right\|_{H^{2}}^{2}+\|\theta(\cdot, t)-\bar{\theta}\|_{H^{1}}^{2} \leq \varepsilon$. Then the solution satisfies

$$
\|\phi(\cdot, t)-\bar{\phi}\|_{H^{2}}+\|\theta(\cdot, t)-\bar{\theta}\|_{H^{1}} \leq \gamma_{2} e^{-\eta_{2} t}, \quad \forall t \geq 0
$$

for some constants $\gamma_{2}, \eta_{2}>0$ which are independent of $t$.

Regarding the above results, we have the following remarks.

REMARK 1.4. The conditions on $F(\cdot)$ are fulfilled for a number of examples, such as $F(z)=\frac{1}{4}\left(z^{2}-1\right)^{2}$ (e.g. [7, 15]). Theorem 1.2 indicates that the non-homogeneous alloy is stable if one of the components is predominant (e.g. when $F(z)=\frac{1}{4}\left(z^{2}-1\right)^{2}$, $F^{\prime \prime}(\cdot) \geq 0$ requires $|\bar{\phi}|>\frac{\sqrt{3}}{3}$ which implies that one of the components is predominant).

REMARK 1.5. It should be pointed out that the decay estimates obtained in the above theorems are not extended to the highest order norm of the solution. However, at the lower level estimates, the main ideas used for the proofs will be fully illustrated. The proof of the decay of the higher order norms is just a tedious long chain of energy estimates which can be easily produced with the help of the lower order estimates. For the simplicity of presentation, we shall not go through the details.

REMARK 1.6. The asymptotic result obtained in Theorem 1.2 has an advantage over the one obtained in Theorem 1.3. Roughly speaking, the former one provides a convenient criterion for determining whether the solution collapses to a constant state or not as time evolves. Based on the result, one only needs to measure the volume of the domain, instead of measuring the "smallness" of the initial perturbation which is usually laborious to perform, to determine whether the solution decays or not when other system parameters are fixed. On the other hand, from the mathematical point of view, we will see in Section 3 that the proof of Theorem 1.3 is more delicate and interesting than that of Theorem 1.2.

REMARK 1.7. It is well-known that the Cahn-Hilliard equation is an effective model in the study of sharp interfaces in two-phase fluid flows. However, based on our results, the order parameter $\phi$ tends to a uniform constant $\bar{\phi}$ instead of \pm 1 . This suggests that under the conditions of Theorem 1.1 and Theorem 1.2, the modeling Equation (1.1) indeed fails to model the sharp interfacial phenomenon. Therefore, our results exhibit some bifurcation phenomena on the effectiveness of the modeling equations.

The plan of the rest of this paper is as follows. In Section 2, we will reformulate the original problem to get an equivalent one for the perturbations, and give a technical lemma which is useful in the estimation of the order parameter. We then prove Theorem 1.2 and Theorem 1.3 in Section 3 and Section 4 respectively.

\section{Reformulation and preliminary}

To study the asymptotic behavior, we first reformulate the original problem to get the new one for the perturbations. For this purpose, we let $\Phi=\phi-\bar{\phi}$ and $\Theta=\theta-\bar{\theta}$, where $\bar{\phi}$ is defined as

$$
\bar{\phi}=\frac{1}{|\Omega|} \int_{\Omega} \phi_{0}(\mathbf{x}) d \mathbf{x}=\frac{1}{|\Omega|} \int_{\Omega} \phi(\mathbf{x}, t) d \mathbf{x}
$$


due to the conservation of total mass and the boundary conditions. Plugging $\Phi$ and $\Theta$ into the original system (1.1) we have

$$
\left\{\begin{array}{l}
\Phi_{t}+U \cdot \nabla \Phi=\nabla \cdot(\beta(\phi) \nabla \mu), \\
\mu=-\alpha \Delta \Phi+F^{\prime}(\phi), \\
U_{t}+U \cdot \nabla U+\nabla \tilde{P}=\mu \nabla \Phi+\Theta \mathbf{e}_{2}, \\
\Theta_{t}+U \cdot \nabla \Theta=\nabla \cdot(\kappa(\phi) \nabla \Theta), \\
\nabla \cdot U=0,
\end{array}\right.
$$

where $\tilde{P}=P-\bar{\theta} y$, and the initial and boundary conditions become

$$
\left\{\begin{array}{l}
(\Phi, \mu, U, \Theta)(\mathbf{x}, 0)=\left(\Phi_{0}, \mu_{0}, U_{0}, \Theta_{0}\right)(\mathbf{x}) \equiv\left(\phi_{0}-\bar{\phi}, \mu_{0}, U_{0}, \theta_{0}-\bar{\theta}\right)(\mathbf{x}), \\
\left.\nabla \Phi \cdot \mathbf{n}\right|_{\partial \Omega}=0,\left.\nabla \mu \cdot \mathbf{n}\right|_{\partial \Omega}=0,\left.U \cdot \mathbf{n}\right|_{\partial \Omega}=0,\left.\Theta\right|_{\partial \Omega}=0 .
\end{array}\right.
$$

Next, we recall the following lemma which is a direct consequence of the Poincaré inequality, whose proof is straightforward.

Lemma 2.1. Let $\Omega$ be any bounded domain in $\mathbb{R}^{n}$ with smooth boundary $\partial \Omega$. Then, for any function $H^{s}(\Omega) \ni f: \Omega \rightarrow \mathbb{R}$, it holds that

(1) $\|f-\bar{f}\|_{H^{2 s}} \leq c\left\|\Delta^{s} f\right\|, \quad\|f-\bar{f}\|_{H^{2 s+1}} \leq c\left\|\nabla \Delta^{s} f\right\|, \quad s \geq 1$, if $\left.\quad \nabla f \cdot \mathbf{n}\right|_{\partial \Omega}=0$;

(2) $\|f\|_{H^{2 s}} \leq c\left\|\Delta^{s} f\right\|, \quad\|f\|_{H^{2 s+1}} \leq c\left\|\nabla \Delta^{s} f\right\|, \quad s \geq 1$, if $\left.f\right|_{\partial \Omega}=0$,

where $\bar{f}=\frac{1}{|\Omega|} \int_{\Omega} f d \mathbf{x}$ and $c=c(s, \Omega)$.

\section{Long-time dynamics for large perturbation}

In this section, we will study the long-time dynamics of classical solutions to (1.1)-(1.2) under the conditions of Theorem 1.2. The proof is based on several steps of careful energy estimates, by exploring the condition $\alpha-F_{3} c_{0}>0$, which are stated as a sequence of lemmas. Since $\beta(\phi)$ is assumed to be a constant, we without loss of generality take $\beta(\phi)=1$. In the next two lemmas we will establish some uniform-intime estimates of the solution, which will be used in the decay estimate. The first one gives the decay estimate of $\Theta$ which will be used to settle down the uniform estimate of $U$.

Lemma 3.1. Under the assumptions of Theorem 1.2, there exists a constant $\gamma_{0}$ independent of $t$ such that for any $t \geq 0$, it holds that

$$
\|\Theta(\cdot, t)\|^{2} \leq\left\|\Theta_{0}\right\|^{2} e^{-2 \gamma_{0} t}, \text { and } \int_{0}^{t}\|\nabla \Theta(\cdot, \tau)\|^{2} e^{\gamma_{0} \tau} d \tau \leq \frac{1}{\kappa_{0}}\left\|\Theta_{0}\right\|^{2} .
$$

Proof. Taking the $L^{2}$ inner product of $(2.1)_{4}$ with $\Theta$ we have

$$
\frac{1}{2} \frac{d}{d t}\|\Theta\|^{2}+\int_{\Omega} \kappa(\phi)|\nabla \Theta|^{2} d \mathbf{x}=0 .
$$

Since $\left.\Theta\right|_{\partial \Omega}=0$, using the condition on $\kappa(\phi)$ and the Poincaré inequality we have

$$
\frac{d}{d t}\|\Theta\|^{2}+\frac{2 \kappa_{0}}{c_{0}}\|\Theta\|^{2} \leq 0
$$


which yields immediately that

$$
\|\Theta(\cdot, t)\|^{2} \leq\left\|\Theta_{0}\right\|^{2} e^{-2 \gamma_{0} t},
$$

where $\gamma_{0}=\kappa_{0} / c_{0}$. This proves the first part of (3.1).

Multiplying (3.2) by $e^{\gamma_{0} t}$ and using (3.4) we get

$$
\frac{d}{d t}\left(e^{\gamma_{0} t}\|\Theta\|^{2}\right)+2 \kappa_{0} e^{\gamma_{0} t}\|\nabla \Theta\|^{2} \leq \gamma_{0} e^{-\gamma_{0} t}\left\|\Theta_{0}\right\|^{2},
$$

which implies the second part of (3.1) immediately after integrating with respect to $t$. This completes the proof of Lemma 3.1.

With the help of Lemma 3.1 we now prove the $H^{1}$ uniform estimate of $U$ and $\Phi$.

Lemma 3.2. Under the conditions of Theorem 1.2, it holds that

$$
\|U(\cdot, t)\|_{H^{1}}^{2}+\|\Phi(\cdot, t)\|_{H^{1}}^{2}+\int_{0}^{t}\|\Phi(\cdot, \tau)\|_{H^{3}}^{2} d \tau \leq c, \quad \forall t \geq 0 .
$$

Proof. Step 1. Taking the $L^{2}$ inner product of $(2.1)_{3}$ with $U$ we have

$$
\frac{1}{2} \frac{d}{d t}\|U\|^{2}=\int_{\Omega} \mu(\nabla \Phi \cdot U) d \mathbf{x}+\int_{\Omega} \Theta \mathbf{e}_{2} \cdot U d \mathbf{x} .
$$

Taking the $L^{2}$ inner product of $(2.1)_{1}$ with $\mu$ we have

$$
\frac{d}{d t}\left(\frac{\alpha}{2}\|\nabla \Phi\|^{2}+\int_{\Omega} F(\phi) d \mathbf{x}\right)+\|\nabla \mu\|^{2}=-\int_{\Omega} \mu(\nabla \Phi \cdot U) d \mathbf{x} .
$$

Adding (3.6) and (3.7) we get

$$
\frac{d}{d t}\left(\frac{1}{2}\|U\|^{2}+\frac{\alpha}{2}\|\nabla \Phi\|^{2}+\int_{\Omega} F(\phi) d \mathbf{x}\right)+\|\nabla \mu\|^{2}=\int_{\Omega} \Theta \mathbf{e}_{2} \cdot U d \mathbf{x}
$$

Applying the Cauchy-Schwarz inequality to the right hand side of (3.8) and using (3.4) we obtain

$$
\frac{d}{d t}\left(\frac{1}{2}\|U\|^{2}+\frac{\alpha}{2}\|\nabla \Phi\|^{2}+\int_{\Omega} F(\phi) d \mathbf{x}\right)+\|\nabla \mu\|^{2} \leq e^{-\gamma_{0} t}\|U\|^{2}+e^{-\gamma_{0} t}\left\|\Theta_{0}\right\|^{2} .
$$

Since $F \geq 0$, Gronwall's inequality then yields

$$
\frac{1}{2}\|U\|^{2}+\frac{\alpha}{2}\|\nabla \Phi\|^{2}+\int_{\Omega} F(\phi) d \mathbf{x}+\int_{0}^{t}\|\nabla \mu(\cdot, \tau)\|^{2} d \tau \leq c, \forall t \geq 0 .
$$

Step 2. We derive some consequences of (3.10). First of all, by using the condition on $F$ and (3.10) we have

$$
\begin{aligned}
\bar{\mu} \equiv \frac{1}{|\Omega|} \int_{\Omega} \mu(\mathbf{x}, t) d \mathbf{x}=\frac{1}{|\Omega|} \int_{\Omega} F^{\prime}(\phi) d \mathbf{x} & \leq \frac{1}{|\Omega|} F_{1}\|\phi\|_{L^{p-1}}^{p-1}+F_{2} \\
& \leq c(p, \Omega)\|\phi\|_{H^{1}}^{p-1}+F_{2} \leq c .
\end{aligned}
$$

Therefore, the average of $\mu$ over the domain remains uniformly bounded for all time. 
Taking the $L^{2}$ inner product of $(\mu-\bar{\mu})$ with $-\Delta \Phi$ we have

$$
\begin{aligned}
-\int_{\Omega}(\mu-\bar{\mu}) \Delta \Phi d \mathbf{x} & =\alpha\|\Delta \Phi\|^{2}+\int_{\Omega} F^{\prime \prime}(\phi)|\nabla \Phi|^{2} d \mathbf{x} \\
& \geq \alpha\|\Delta \Phi\|^{2}-F_{3}\|\nabla \Phi\|^{2} .
\end{aligned}
$$

Using the boundary conditions and the Cauchy-Schwarz and Poincaré inequalities we have

$$
\|\nabla \Phi\|^{2}=-\int_{\Omega} \Phi \Delta \Phi d \mathbf{x} \leq \frac{1}{2 c_{0}}\|\Phi\|^{2}+\frac{c_{0}}{2}\|\Delta \Phi\|^{2} \leq \frac{1}{2}\|\nabla \Phi\|^{2}+\frac{c_{0}}{2}\|\Delta \Phi\|^{2},
$$

which implies that

$$
\|\nabla \Phi\|^{2} \leq c_{0}\|\Delta \Phi\|^{2}
$$

where $c_{0}$ is the constant in the Poincaré inequality on the domain $\Omega$. Under the condition $\alpha_{1} \equiv \alpha-F_{3} c_{0}>0$, by (3.11) we have

$$
-\int_{\Omega}(\mu-\bar{\mu}) \Delta \Phi d \mathbf{x} \geq \alpha_{1}\|\Delta \Phi\|^{2} .
$$

On the other hand, by the Cauchy-Schwarz inequality we have

$$
-\int_{\Omega}(\mu-\bar{\mu}) \Delta \Phi d \mathbf{x} \leq \frac{1}{2 \alpha_{1}}\|\mu-\bar{\mu}\|^{2}+\frac{\alpha_{1}}{2}\|\Delta \Phi\|^{2} .
$$

Hence, it holds that

$$
\|\Delta \Phi\|^{2} \leq \frac{1}{\alpha_{1}^{2}}\|\mu-\bar{\mu}\|^{2} \leq \frac{c_{0}}{\alpha_{1}^{2}}\|\nabla \mu\|^{2},
$$

which implies, by (3.10), that

$$
\int_{0}^{t}\|\Delta \Phi(\cdot, \tau)\|^{2} d \tau \leq c
$$

Step 3. We observe, by definition of $\Phi$, Lemma 2.1 , and $(2.1)_{2}$, that

$$
\|\Phi\|_{H^{3}}^{2} \leq c\|\nabla(\Delta \Phi)\|^{2} \leq c\left(\|\nabla \mu\|^{2}+\left\|F^{\prime \prime}(\phi) \nabla \Phi\right\|^{2}\right) .
$$

By Hölder's inequality, Lemma 2.1, and (3.10) we have

$$
\left\|F^{\prime \prime}(\phi) \nabla \Phi\right\|^{2} \leq c\left(\|\phi\|_{L^{4(p-2)}}^{2(p-2)}\|\nabla \Phi\|_{L^{4}}^{2}+\|\nabla \Phi\|^{2}\right) \leq c\|\Delta \Phi\|^{2},
$$

where we have used the Sobolev inequality $\|f\|_{L^{p}} \leq c(p)\|f\|_{H^{1}}$ in 2D. Plugging (3.14) into (3.13) we have

$$
\|\Phi\|_{H^{3}}^{2} \leq c\left(\|\nabla \mu\|^{2}+\|\Delta \Phi\|^{2}\right),
$$

which, together with (3.12) and (3.10), implies that

$$
\int_{0}^{t}\|\Phi(\cdot, \tau)\|_{H^{3}}^{2} d \tau \leq c, \quad \forall t \geq 0 .
$$


Step 4. By taking the curl of the velocity equation we get

$$
\omega_{t}+U \cdot \nabla \omega=\mu_{x} \Phi_{y}-\mu_{y} \Phi_{x}+\Theta_{x}
$$

where $\omega=v_{x}-u_{y}$ is the $2 \mathrm{D}$ vorticity. Taking the $L^{2}$ inner product of (3.17) with $\omega$ and applying Hölder's inequality we have

$$
\frac{1}{2} \frac{d}{d t}\|\omega\|^{2} \leq\left(\left\|\mu_{x} \Phi_{y}-\mu_{y} \Phi_{x}\right\|+\left\|\Theta_{x}\right\|\right)\|\omega\|,
$$

which implies that

$$
\frac{d}{d t}\|\omega\| \leq 2\|\nabla \mu\|\|\nabla \Phi\|_{L^{\infty}}+\|\nabla \Theta\| .
$$

Upon integrating (3.18) with respect to $t$ and using Hölder and Sobolev inequalities we have

$$
\begin{aligned}
\|\omega(\cdot, t)\| \leq & \int_{0}^{t}\left(2\|\nabla \mu\|\|\nabla \Phi\|_{L^{\infty}}+\|\nabla \Theta\|\right) d \tau+\left\|\omega_{0}\right\| \\
\leq & 2\left(\int_{0}^{t}\|\nabla \mu\|^{2} d \tau\right)^{\frac{1}{2}}\left(\int_{0}^{t}\|\Phi\|_{H^{3}}^{2} d \tau\right)^{\frac{1}{2}} \\
& +\left(\int_{0}^{t} e^{\gamma_{0} \tau / 2}\|\nabla \Theta\|^{2} d \tau\right)^{\frac{1}{2}}\left(\int_{0}^{t} e^{-\gamma_{0} \tau / 2} d \tau\right)^{\frac{1}{2}}+\left\|\omega_{0}\right\| .
\end{aligned}
$$

From previous estimates (c.f. (3.4), (3.10), (3.16)) we know that the RHS of (3.19) is uniformly bounded in time. Therefore, we have $\|\omega(\cdot, t)\| \leq c, \forall t \geq 0$, which, together with (3.10) and (3.16), implies (3.5). This completes the proof of Lemma 3.2.

Using the uniform estimates obtained in Lemma 3.2 we prove the exponential decay of $\|\Phi(t)\|_{H^{2}}^{2}$.

Lemma 3.3. Under the conditions of Theorem 1.2, there exist constants $c_{1}, c_{2}>0$ independent of $t$ such that

$$
\|\Phi(t)\|_{H^{2}}^{2} \leq c_{1} e^{-c_{2} t}, \quad \forall t \geq 0 .
$$

Proof. Step 1. Taking the $L^{2}$ inner product of $(2.1)_{1}$ with $\Delta \Phi$ and integrating by parts we get

$$
\begin{aligned}
\frac{1}{2} \frac{d}{d t}\|\nabla \Phi\|^{2}+\alpha\|\nabla \Delta \Phi\|^{2} & =-\int_{\Omega} \Phi U \cdot(\nabla \Delta \Phi) d \mathbf{x}+\int_{\Omega} \nabla F^{\prime}(\phi) \cdot \nabla \Delta \Phi d \mathbf{x} \\
& \leq \frac{\alpha}{2}\|\nabla \Delta \Phi\|^{2}+\frac{1}{\alpha}\|\Phi U\|^{2}+\frac{1}{\alpha}\left\|\nabla F^{\prime}(\phi)\right\|^{2} \\
& \leq \frac{\alpha}{2}\|\nabla \Delta \Phi\|^{2}+\frac{1}{\alpha}\|U\|^{2}\|\Phi\|_{L^{\infty}}^{2}+\frac{1}{\alpha}\left\|F^{\prime \prime}(\phi)\right\|_{L^{4}}^{2}\|\nabla \Phi\|_{L^{4}}^{2} \\
& \leq \frac{\alpha}{2}\|\nabla \Delta \Phi\|^{2}+c\|\Delta \Phi\|^{2},
\end{aligned}
$$

where we have used Lemma 3.2, the Sobolev embeddings $H^{2} \hookrightarrow L^{\infty}$ and $H^{1} \hookrightarrow L^{4}$ in 2D, and Lemma 2.2. This gives

$$
\frac{d}{d t}\|\nabla \Phi\|^{2}+\alpha\|\nabla \Delta \Phi\|^{2} \leq c\|\Delta \Phi\|^{2} .
$$


Step 2. Taking the $L^{2}$ inner product of $(2.1)_{1}$ with $\Delta^{2} \Phi$ and using the CauchySchwarz inequality we get

$$
\begin{aligned}
& \frac{d}{d t}\|\Delta \Phi\|^{2}+\alpha\left\|\Delta^{2} \Phi\right\|^{2} \\
\leq & \frac{2}{\alpha}\|U \cdot \nabla \Phi\|^{2}+\frac{2}{\alpha}\left\|\Delta F^{\prime}(\phi)\right\|^{2} \\
\leq & \frac{2}{\alpha}\|U\|^{2}\|\nabla \Phi\|_{L^{\infty}}^{2}+c\left(\left\|F^{\prime \prime}(\phi)\right\|_{L^{4}}^{2}\|\Delta \Phi\|_{L^{4}}^{2}+\left\|F^{\prime \prime \prime}(\phi)\right\|^{2}\|\nabla \Phi\|_{L^{\infty}}^{4}\right) \\
\leq & c\|\nabla \Delta \Phi\|^{2}+c\|\nabla \Delta \Phi\|^{2}+c\|\nabla \Phi\|^{2}\|\nabla \Delta \Phi\|^{2} \\
\leq & c\|\nabla \Delta \Phi\|^{2},
\end{aligned}
$$

where we have used the interpolation inequality $\|f\|_{L^{\infty}} \leq c\|f\|_{H^{2}}^{1 / 2}\|f\|_{L^{2}}^{1 / 2}$ in $2 \mathrm{D}$. This gives

$$
\frac{d}{d t}\|\Delta \Phi\|^{2}+\alpha\left\|\Delta^{2} \Phi\right\|^{2} \leq c\|\nabla \Delta \Phi\|^{2} .
$$

Coupling (3.20) and (3.21) we have

$$
\frac{d}{d t}\left(c(\alpha)\|\nabla \Phi\|^{2}+\|\Delta \Phi\|^{2}\right)+c(\alpha)\|\nabla \Delta \Phi\|^{2}+\alpha\left\|\Delta^{2} \Phi\right\|^{2} \leq c\|\Delta \Phi\|^{2} .
$$

Step 3. Taking the $L^{2}$ inner product of $(2.1)_{1}$ with $\Phi$, after integrating by parts we have

$$
\frac{1}{2} \frac{d}{d t}\|\Phi\|^{2}=\int_{\Omega} \Phi \Delta \mu d \mathbf{x}=-\int_{\Omega} \nabla \mu \cdot \nabla \Phi d \mathbf{x}=-\alpha\|\Delta \Phi\|^{2}-\int_{\Omega} F^{\prime \prime}(\phi)|\nabla \Phi|^{2} d \mathbf{x},
$$

which gives

$$
\frac{1}{2} \frac{d}{d t}\|\Phi\|^{2}+\alpha\|\Delta \Phi\|^{2} \leq F_{3}\|\nabla \Phi\|^{2} .
$$

It turns out that

$$
\frac{1}{2} \frac{d}{d t}\|\Phi\|^{2}+\alpha_{1}\|\Delta \Phi\|^{2} \leq 0
$$

Using (3.24) to absorb the right hand side of (3.22) we arrive at

$$
\begin{aligned}
\frac{d}{d t}\left[c(\alpha)\|\Phi\|^{2}+c(\alpha)\|\nabla \Phi\|^{2}+\right. & \left.\|\Delta \Phi\|^{2}\right] \\
& +c(\alpha)\|\nabla \Delta \Phi\|^{2}+\alpha\left\|\Delta^{2} \Phi\right\|^{2}+c\left(\alpha_{1}\right)\|\Delta \Phi\|^{2} \leq 0,
\end{aligned}
$$

which yields the exponential decay of $\|\Phi(t)\|_{H^{2}}^{2}$ immediately with the help of Lemma 2.1. This completes the proof of Lemma 3.3.

We finally show the decay of $\|\Theta(t)\|_{H^{1}}^{2}$.

Lemma 3.4. There exist constants $c_{3}, c_{4}>0$ independent of $t$ such that

$$
\|\Theta(t)\|_{H^{1}}^{2} \leq c_{3} e^{-c_{4} t}, \quad \forall t \geq 0 .
$$


Proof. Taking the $L^{2}$ inner product of $(2.1)_{4}$ with $\Delta \Theta$ we have

$$
\begin{aligned}
& \frac{1}{2} \frac{d}{d t}\|\nabla \Theta\|^{2}+\int_{\Omega} \kappa(\phi)|\Delta \Theta|^{2} d \mathbf{x} \\
= & \int_{\Omega}(U \cdot \nabla \Theta) \Delta \Theta d \mathbf{x}-\int_{\Omega}\left(\kappa^{\prime}(\phi) \nabla \Phi \cdot \nabla \Theta\right) \Delta \Theta d \mathbf{x} \\
\leq & \frac{\kappa_{0}}{4}\|\Delta \Theta\|^{2}+c\|U\|_{L^{4}}^{2}\|\nabla \Theta\|_{L^{4}}^{2}+c\|\nabla \Phi\|_{L^{4}}^{2}\|\nabla \Theta\|_{L^{4}}^{2} \\
\leq & \frac{\kappa_{0}}{4}\|\Delta \Theta\|^{2}+c\left(\|U\|_{H^{1}}^{2}+\|\nabla \Phi\|_{H^{1}}^{2}\right)\left(\|\nabla \Theta\|\|\Delta \Theta\|+\|\nabla \Theta\|^{2}\right) \\
\leq & \frac{\kappa_{0}}{2}\|\Delta \Theta\|^{2}+c\|\nabla \Theta\|^{2} .
\end{aligned}
$$

where we have used Lemma 3.2, Lemma 3.3, and the Gagliardo-Nirenberg type inequality $\|f\|_{L^{4}}^{2} \leq c\left(\|f\|\|\nabla f\|+\|f\|^{2}\right)$ in $2 \mathrm{D}$. This gives

$$
\frac{d}{d t}\|\nabla \Theta\|^{2}+\kappa_{0}\|\Delta \Theta\|^{2} \leq c\|\nabla \Theta\|^{2} .
$$

By coupling (3.2) with (3.26) we conclude that

$$
\frac{d}{d t}\left(c\|\Theta\|^{2}+2\|\nabla \Theta\|^{2}\right)+\|\Delta \Theta\|^{2}+c\|\nabla \Theta\|^{2} \leq 0,
$$

which gives the exponential decay of $\|\Theta(t)\|_{H^{1}}^{2}$. This completes the proof of Lemma 3.4 .

REMARK 3.5. Since the problem is set on the bounded domain, which distinguishes it from the Cauchy problem, the spatial derivatives of the solution are unknown on the boundary of the domain. However, by working on the temporal derivatives of $\Phi$ and $\Theta$ and using an iteration argument one can show the exponential decay rate of higher order norms of the solution with the aid of the lower order estimates. We omit the details here.

\section{Long-time dynamics for small perturbations}

In this section, we study a different aspect of the long-time dynamics of the solution to (1.1)-(1.2). Instead of assuming the threshold value of the diffuse interface parameter $\alpha$, we study the asymptotic behavior of (1.1) under the smallness assumption on the initial perturbation.

Step 1. First of all, without loss of generality we assume that $\nu=\bar{\phi}=$ $\frac{1}{|\Omega|} \int_{\Omega} \phi_{0}(\mathbf{x}) d \mathbf{x}>0$, and assume that for some fixed $0<\eta<\bar{\phi}$, it holds that $F^{\prime \prime}(\cdot) \geq$ 0 on $I_{\eta}=[\nu-\eta, \nu+\eta]$.

Second, one can easily construct a function $F_{\nu}(\cdot)$ such that

$$
F_{\nu}(\nu)=0, \quad F_{\nu}^{\prime}(\nu)=0, \quad F_{\nu}^{\prime \prime}(z)=F^{\prime \prime}(z) \text { on } I_{\eta}, \quad F_{\nu}^{\prime \prime}(z) \geq 0 \text { if } z \notin I_{\eta},
$$

and such that all of the conditions imposed on $F(\cdot)$ indicated in Theorem 1.1 are fulfilled by $F_{\nu}(\cdot)$. By construction, it is easy to see that $F_{\nu} \geq 0$. By the convexity of $F_{\nu}$ we see that $F_{\nu}(\nu)-F_{\nu}(z) \geq F_{\nu}^{\prime}(z)(\nu-z)$, which, together with (4.1), implies that

$$
F_{\nu}(z) \leq F_{\nu}^{\prime}(z)(z-\nu), \quad \forall z \in \mathbb{R} .
$$

In particular, there exist positive constants $F_{4}$ and $F_{5}$ such that (c.f. Theorem 1.1)

$$
\left|F_{\nu}^{\prime}(z)\right| \leq F_{4}+F_{5}|z|^{p-1}, \quad\left|F_{\nu}^{\prime \prime}(z)\right| \leq F_{4}+F_{5}|z|^{p-2}, \quad \forall z \in \mathbb{R} \text {, for some } p \geq 6 .
$$


Step 2. Next, we solve the original problem with $F$ replaced by $F_{\nu}$ and with the same initial data, and denote the solution by $\Phi_{\nu}, \mu_{\nu}, U_{\nu}$, and $\Theta_{\nu}$. In this situation, the estimate (3.9) becomes

$$
\begin{aligned}
& \frac{d}{d t}\left(\frac{1}{2}\left\|U_{\nu}\right\|^{2}+\frac{\alpha}{2}\left\|\nabla \Phi_{\nu}\right\|^{2}+\left\|F_{\nu}\left(\phi_{\nu}\right)\right\|_{L^{1}}\right)+\int_{\Omega} \beta(\phi)\left|\nabla \mu_{\nu}\right|^{2} d \mathbf{x} \\
\leq & e^{-\gamma_{0} t}\left\|U_{\nu}\right\|^{2}+e^{-\gamma_{0} t}\left\|\Theta_{0}\right\|^{2} .
\end{aligned}
$$

Using the definition of $\nu$ and the conservation of total mass we have

$$
\begin{aligned}
& \frac{d}{d t}\left(\frac{1}{2}\left\|U_{\nu}\right\|^{2}+\frac{\alpha}{2}\left\|\nabla \Phi_{\nu}\right\|^{2}+\left\|G\left(\phi_{\nu}, \nu\right)\right\|_{L^{1}}\right)+\int_{\Omega} \beta\left(\phi_{\nu}\right)\left|\nabla \mu_{\nu}\right|^{2} d \mathbf{x} \\
\leq & e^{-\gamma_{0} t}\left\|U_{\nu}\right\|^{2}+e^{-\gamma_{0} t}\left\|\Theta_{0}\right\|^{2},
\end{aligned}
$$

where $G\left(\phi_{\nu}, \nu\right)=F_{\nu}\left(\phi_{\nu}\right)-F_{\nu}(\nu)-F_{\nu}^{\prime}(\nu)\left(\phi_{\nu}-\nu\right)$ satisfying $G\left(\phi_{\nu}, \nu\right) \geq 0$ due to $F_{\nu}^{\prime \prime}(\cdot) \geq$ 0 . By Gronwall's inequality we have

$$
\begin{aligned}
& \frac{1}{2}\left\|U_{\nu}\right\|^{2}+\frac{\alpha}{2}\left\|\nabla \Phi_{\nu}\right\|^{2}+\left\|G\left(\phi_{\nu}, \nu\right)\right\|_{L^{1}}+\int_{0}^{t} \beta_{0}\left\|\nabla \mu_{\nu}\right\|^{2} d \tau \\
\leq & c\left(\left\|U_{0}\right\|^{2}+\left\|\nabla \Phi_{0}\right\|^{2}+\left\|G\left(\phi_{0}, \nu\right)\right\|_{L^{1}}+\left\|\Theta_{0}\right\|^{2}\right),
\end{aligned}
$$

where we have used the condition $\beta_{0} \leq \beta(\phi)$. Since $G\left(\phi_{0}, \nu\right)=F^{\prime \prime}(\xi)\left(\phi_{0}-\nu\right)^{2}$ for some $\xi$ between $\phi_{0}$ and $\nu$, by (4.3) we have

$$
\begin{aligned}
\left\|G\left(\phi_{0}, \nu\right)\right\|_{L^{1}} & \leq \int_{\Omega}\left(F_{4}+F_{5}|\xi|^{p-2}\right)\left(\phi_{0}-\nu\right)^{2} d \mathbf{x} \\
& \leq c\left(\left\|\phi_{0}\right\|_{L^{\infty}}^{p-2}+|\nu|^{p-2}+1\right)\left\|\phi_{0}-\nu\right\|^{2} \\
& \leq c\left(\left\|\phi_{0}\right\|_{H^{2}}^{p-2}+|\nu|^{p-2}+1\right)\left\|\Phi_{0}\right\|^{2} .
\end{aligned}
$$

So we update (4.5) as

$$
\begin{aligned}
& \frac{1}{2}\left\|U_{\nu}\right\|^{2}+\frac{\alpha}{2}\left\|\nabla \Phi_{\nu}\right\|^{2}+\left\|G\left(\phi_{\nu}, \nu\right)\right\|_{L^{1}}+\int_{0}^{t} \beta_{0}\left\|\nabla \mu_{\nu}\right\|^{2} d \tau \\
\leq & c\left(\left\|U_{0}\right\|^{2}+\left\|\Phi_{0}\right\|_{H^{1}}^{2}+\left\|\Theta_{0}\right\|^{2}\right) .
\end{aligned}
$$

Step 3. In this step, we will exploit the estimate (4.6) to get more information about the auxiliary solution. Taking the $L^{2}$ inner product of $\mu_{\nu}$ with $-\Delta \Phi_{\nu}$ and noticing that $F_{\nu}^{\prime \prime} \geq 0$ we have

$$
\left(\mu_{\nu},-\Delta \Phi_{\nu}\right)=\alpha\left\|\Delta \Phi_{\nu}\right\|^{2}+\int_{\Omega} F_{\nu}^{\prime \prime}\left(\phi_{\nu}\right)\left|\nabla \Phi_{\nu}\right|^{2} d \mathbf{x} \geq \alpha\left\|\Delta \Phi_{\nu}\right\|^{2} .
$$

On the other hand, since $\left(\bar{\mu}_{\nu},-\Delta \Phi_{\nu}\right)=0$, where $\bar{\mu}_{\nu}=\frac{1}{|\Omega|} \int_{\Omega} \mu_{\nu}(\mathbf{x}, t) d \mathbf{x}$, we have

$$
\left(\mu_{\nu},-\Delta \Phi_{\nu}\right)=\left(\mu_{\nu}-\bar{\mu}_{\nu},-\Delta \Phi_{\nu}\right) \leq\left\|\mu-\bar{\mu}_{\nu}\right\|\left\|\Delta \Phi_{\nu}\right\| \leq \frac{\alpha}{2}\left\|\Delta \Phi_{\nu}\right\|^{2}+c\left\|\nabla \mu_{\nu}\right\|^{2} .
$$

Combining (4.7) with (4.8) we get

$$
\left\|\nabla \mu_{\nu}\right\|^{2} \geq c\left\|\Delta \Phi_{\nu}\right\|^{2}
$$


Therefore, plugging (4.9) into (4.6) we have for any $t \geq 0$

$$
\begin{aligned}
& \left\|U_{\nu}\right\|^{2}+\left\|\nabla \Phi_{\nu}\right\|^{2}+\left\|G\left(\phi_{\nu}, \nu\right)\right\|_{L^{1}}+\int_{0}^{t}\left(\left\|\nabla \mu_{\nu}\right\|^{2}+\left\|\Delta \Phi_{\nu}\right\|^{2}\right) d \tau \\
\leq & c\left(\left\|U_{0}\right\|^{2}+\left\|\Phi_{0}\right\|_{H^{1}}^{2}+\left\|\Theta_{0}\right\|^{2}\right) .
\end{aligned}
$$

Step 4. Since the solution exists globally in 2D, from (4.10) we know that when $\left\|U_{0}\right\|^{2}+\left\|\Phi_{0}\right\|_{H^{2}}^{2}+\left\|\Theta_{0}\right\|^{2}$ is sufficiently small, for any $t \geq 0$ it holds that

$$
\int_{0}^{t}\left(\left\|\nabla \mu_{\nu}\right\|^{2}+\left\|\Delta \Phi_{\nu}\right\|^{2}\right) d \tau \leq \varepsilon_{1} .
$$

By (3.15) we have

$$
\left\|\nabla \Delta \Phi_{\nu}\right\|^{2} \leq c\left(\left\|\nabla \mu_{\nu}\right\|^{2}+\left\|\Delta \Phi_{\nu}\right\|^{2}\right),
$$

which, together with (4.11), implies that

$$
\int_{0}^{t}\left\|\nabla \Delta \Phi_{\nu}\right\|^{2} d \tau \leq \varepsilon_{2}, \quad \forall t \geq 0
$$

Step 5. Taking the $L^{2}$ inner product of $(2.1)_{1}$ with $\Delta^{2} \Phi_{\nu}$ we have

$$
\begin{aligned}
& \frac{1}{2} \frac{d}{d t}\left\|\Delta \Phi_{\nu}\right\|^{2}+\alpha \int_{\Omega} \beta(\phi)\left|\Delta^{2} \Phi_{\nu}\right|^{2} d \mathbf{x} \\
= & \int_{\Omega}\left(\beta\left(\phi_{\nu}\right) \Delta F_{\nu}^{\prime}\left(\phi_{\nu}\right)+\beta^{\prime}\left(\phi_{\nu}\right) \nabla \Phi_{\nu} \cdot\left(\nabla \Delta \Phi_{\nu}+\nabla F_{\nu}^{\prime}\left(\phi_{\nu}\right)\right)-U_{\nu} \cdot \nabla \Phi_{\nu}\right) \Delta^{2} \Phi_{\nu} d \mathbf{x} .
\end{aligned}
$$

We estimate the right hand side term by term. First of all, using the condition on $\beta(\phi)$ we have

$$
\begin{aligned}
\int_{\Omega} \beta\left(\phi_{\nu}\right) \Delta F_{\nu}^{\prime}\left(\phi_{\nu}\right) \Delta^{2} \Phi_{\nu} d \mathbf{x} & \leq \delta\left\|\Delta^{2} \Phi_{\nu}\right\|^{2}+c(\delta)\left\|\Delta F_{\nu}^{\prime}\left(\phi_{\nu}\right)\right\|^{2} \\
& \leq \delta\left\|\Delta^{2} \Phi_{\nu}\right\|^{2}+c(\delta)\left\|\nabla \Delta \Phi_{\nu}\right\|^{2}
\end{aligned}
$$

where $\delta>0$ is a constant to be determined.

Second, using (4.10) and the interpolation inequality $\|f\|_{L^{4}}^{2} \leq c\left(\|f\|\|\nabla f\|+\|f\|^{2}\right)$ in $2 \mathrm{D}$ we have

$$
\begin{aligned}
& \int_{\Omega} \beta^{\prime}\left(\phi_{\nu}\right)\left(\nabla \Phi_{\nu} \cdot \nabla \Delta \Phi_{\nu}\right) \Delta^{2} \Phi_{\nu} d \mathbf{x} \\
\leq & \frac{\delta}{2}\left\|\Delta^{2} \Phi_{\nu}\right\|^{2}+c(\delta)\left\|\nabla \Phi_{\nu}\right\|_{L^{4}}^{2}\left\|\nabla \Delta \Phi_{\nu}\right\|_{L^{4}}^{2} \\
\leq & \frac{\delta}{2}\left\|\Delta^{2} \Phi_{\nu}\right\|^{2}+c(\delta)\left(\left\|\nabla \Phi_{\nu}\right\|\left\|\Delta \Phi_{\nu}\right\|+\left\|\nabla \Phi_{\nu}\right\|\right)\left(\left\|\nabla \Delta \Phi_{\nu}\right\|\left\|\Delta^{2} \Phi_{\nu}\right\|+\left\|\nabla \Delta \Phi_{\nu}\right\|^{2}\right) \\
\leq & \frac{\delta}{2}\left\|\Delta^{2} \Phi_{\nu}\right\|^{2}+c(\delta)\left(\left\|\Delta \Phi_{\nu}\right\|+1\right)\left(\left\|\nabla \Delta \Phi_{\nu}\right\|\left\|\Delta^{2} \Phi_{\nu}\right\|+\left\|\nabla \Delta \Phi_{\nu}\right\|^{2}\right) \\
\leq & \delta\left\|\Delta^{2} \Phi_{\nu}\right\|^{2}+c(\delta)\left\|\Delta \Phi_{\nu}\right\|^{2}\left\|\nabla \Delta \Phi_{\nu}\right\|^{2} .
\end{aligned}
$$

Third, by using the condition on $F_{\nu}$ and (4.10) we have

$$
\int_{\Omega} \beta^{\prime}\left(\phi_{\nu}\right)\left(\nabla \Phi_{\nu} \cdot \nabla F_{\nu}^{\prime}\left(\phi_{\nu}\right)\right) \Delta^{2} \Phi_{\nu} d \mathbf{x} \leq \delta\left\|\Delta^{2} \Phi_{\nu}\right\|^{2}+c(\delta)\left\|\nabla \Phi_{\nu} \cdot \nabla F_{\nu}^{\prime}\left(\phi_{\nu}\right)\right\|^{2}
$$




$$
\begin{aligned}
& \leq \delta\left\|\Delta^{2} \Phi_{\nu}\right\|^{2}+c(\delta)\left\|\nabla \Phi_{\nu}\right\|_{L^{\infty}}^{4}\left\|F_{\nu}^{\prime \prime}\left(\phi_{\nu}\right)\right\|^{2} \\
& \leq \delta\left\|\Delta^{2} \Phi_{\nu}\right\|^{2}+c(\delta)\left\|\nabla \Phi_{\nu}\right\|_{H^{1}}^{2}\left\|\nabla \Phi_{\nu}\right\|_{H^{2}}^{2} \\
& \leq \delta\left\|\Delta^{2} \Phi_{\nu}\right\|^{2}+c(\delta)\left\|\nabla \Delta \Phi_{\nu}\right\|^{2}\left\|\Delta \Phi_{\nu}\right\|^{2}
\end{aligned}
$$

where we have used the interpolation inequality $\|f\|_{L^{\infty}}^{2} \leq c\|f\|_{H^{1}}\|f\|_{H^{2}}$ in $2 \mathrm{D}$ and Lemma 2.1.

Fourth, using (4.10) we have

$$
\begin{aligned}
\int_{\Omega}\left(U_{\nu} \cdot \nabla \Phi_{\nu}\right) \Delta^{2} \Phi_{\nu} d \mathbf{x} & \leq \delta\left\|\Delta^{2} \Phi_{\nu}\right\|^{2}+c(\delta)\left\|U_{\nu} \cdot \nabla \Phi_{\nu}\right\|^{2} \\
& \leq \delta\left\|\Delta^{2} \Phi_{\nu}\right\|^{2}+c(\delta)\left\|U_{\nu}\right\|^{2}\left\|\nabla \Phi_{\nu}\right\|_{L^{\infty}}^{2} \\
& \leq \delta\left\|\Delta^{2} \Phi_{\nu}\right\|^{2}+c(\delta)\left\|\nabla \Delta \Phi_{\nu}\right\|^{2} .
\end{aligned}
$$

Choosing $\delta=\alpha \beta_{0} / 8$ and plugging (4.15)-(4.18) into (4.14), after rearranging terms we get

$$
\frac{d}{d t}\left\|\Delta \Phi_{\nu}\right\|^{2}+\alpha \beta_{0}\left\|\Delta^{2} \Phi_{\nu}\right\|^{2} \leq c\left\|\Delta \Phi_{\nu}\right\|^{2}\left\|\nabla \Delta \Phi_{\nu}\right\|^{2}+c\left\|\nabla \Delta \Phi_{\nu}\right\|^{2} .
$$

Applying Gronwall's inequality to (4.19), using (4.13) and the smallness of the initial data we have

$$
\left\|\Delta \Phi_{\nu}\right\|^{2}+\int_{0}^{t}\left\|\Delta^{2} \Phi_{\nu}\right\|^{2} d \tau \leq \varepsilon_{3}, \quad \forall t \geq 0,
$$

which implies that (c.f. Lemma 2.1)

$$
\left\|\Phi_{\nu}\right\|_{H^{2}}^{2} \leq \varepsilon_{4}, \quad \forall t \geq 0 .
$$

By the Sobolev embedding $H^{2} \hookrightarrow C^{0}$ in $2 \mathrm{D}$ we then have

$$
\left\|\phi_{\nu}-\nu\right\|_{C^{0}}^{2} \leq \varepsilon_{5}, \quad \forall t \geq 0 .
$$

We remark that the constants $\varepsilon_{i}(i=1, \cdots, 5)$ are independent of time and will go to zero as $\left\|U_{0}\right\|^{2}+\left\|\Phi_{0}\right\|_{H^{2}}^{2}+\left\|\Theta_{0}\right\|^{2}$ tends to zero.

Step 6. We observe from (4.22) that, when $\left\|U_{0}\right\|^{2}+\left\|\Phi_{0}\right\|_{H^{2}}^{2}+\left\|\Theta_{0}\right\|^{2}$ is sufficiently small, $\phi_{\nu} \in I_{\eta}=[\nu-\eta, \nu+\eta]$ for all $t \geq 0$. By (4.1) we know that

$$
F_{\nu}(z)=F(z)-F(\nu)-(z-\nu) F^{\prime}(\nu) \text { on } I_{\eta} .
$$

Therefore, $\left(\phi_{\nu}, \mu_{\nu}, U_{\nu}, \theta_{\nu}\right)$ is indeed a global classical solution of (1.1)-(1.2) with $F(z)$ replaced by $F(z)-F(\nu)-(z-\nu) F^{\prime}(\nu)$. From the definition of $\mu$ and the first equation of (1.1) we see that adding an affine function to $F$ does not change the equations. Thus, by the uniqueness of classical solutions we know that $\phi_{\nu}=\phi$ for all $t \geq 0$, when $\left\|U_{0}\right\|^{2}+\left\|\Phi_{0}\right\|_{H^{2}}^{2}+\left\|\Theta_{0}\right\|^{2}$ is sufficiently small. Hence, $\phi \in I_{\eta}$ for all $t \geq 0$, which in turn gives $F^{\prime \prime}(\phi) \geq 0$ for all $t \geq 0$ by assumption.

Step 7. Taking the $L^{2}$ inner product of $(2.1)_{1}$ with $\Phi$ we have

$$
\begin{aligned}
& \frac{1}{2} \frac{d}{d t}\|\Phi\|^{2}+\alpha \int_{\Omega} \beta(\phi)|\Delta \Phi|^{2} d \mathbf{x} \\
= & -\alpha \int_{\Omega} \beta^{\prime}(\phi) \Delta \Phi|\nabla \Phi|^{2} d \mathbf{x}-\int_{\Omega} \beta(\phi) F^{\prime \prime}(\phi)|\nabla \Phi|^{2} d \mathbf{x}
\end{aligned}
$$




$$
\leq-\alpha \int_{\Omega} \beta^{\prime}(\phi) \Delta \Phi|\nabla \Phi|^{2} d \mathbf{x}
$$

where we used the fact that $F^{\prime \prime}(\phi) \geq 0$. Using the Cauchy-Schwarz inequality we have

$$
\begin{aligned}
-\alpha \int_{\Omega} \beta^{\prime}(\phi) \Delta \Phi|\nabla \Phi|^{2} d \mathbf{x} & \leq \frac{\alpha \beta_{0}}{2}\|\Delta \Phi\|^{2}+c\|\nabla \Phi\|_{L^{4}}^{4} \\
& \leq \frac{\alpha \beta_{0}}{2}\|\Delta \Phi\|^{2}+c\|\nabla \Phi\|^{2}\|\Delta \Phi\|^{2} .
\end{aligned}
$$

Combining (4.24) and (4.25) we get

$$
\frac{d}{d t}\|\Phi\|^{2}+\alpha \beta_{0}\|\Delta \Phi\|^{2} \leq c\|\nabla \Phi\|^{2}\|\Delta \Phi\|^{2} .
$$

Since $F^{\prime \prime} \geq 0$, we know that (4.6) is valid for $\Phi$. Therefore, when $\left\|U_{0}\right\|^{2}+\left\|\Phi_{0}\right\|_{H^{2}}^{2}+$ $\left\|\Theta_{0}\right\|^{2}$ is small enough, we see that $\|\nabla \Phi\|^{2}$ is sufficiently small. Whence, we get from (4.26) that

$$
\frac{d}{d t}\|\Phi\|^{2}+\frac{\alpha \beta_{0}}{2}\|\Delta \Phi\|^{2} \leq 0
$$

By taking the $L^{2}$ inner product of $(2.1)_{1}$ with $\Delta \Phi$ one can get a similar estimate as $(3.20)$ :

$$
\frac{d}{d t}\|\nabla \Phi\|^{2}+\alpha \beta_{0}\|\nabla \Delta \Phi\|^{2} \leq c\|\Delta \Phi\|^{2}
$$

Since (4.19) is also valid for $\Phi$, by coupling (4.27), (4.28), and (4.19) and using the smallness of $\left\|U_{0}\right\|^{2}+\left\|\Phi_{0}\right\|_{H^{2}}^{2}+\left\|\Theta_{0}\right\|^{2}$ one can get a similar estimate as (3.25), which implies the exponential decay of $\|\Phi(t)\|_{H^{2}}^{2}$. The decay of $\|\Theta(t)\|_{H^{1}}^{2}$ in this case can be proved in a similar fashion as in Lemma 3.4. We omit the details. This completes the proof of Theorem 1.3.

We finish this section with the following remarks.

REMARK 4.1. We observe that using the arguments in this paper one can show that the results obtained in Theorem 1.2 and Theorem 1.3 still hold if the Dirichlet boundary condition for $\theta$ is replaced by the Neumann boundary condition $\left.\nabla \theta \cdot \mathbf{n}\right|_{\partial \Omega}=$ 0 . In this case, the equilibrium state of $\theta$ is $\tilde{\theta}=\frac{1}{|\Omega|} \int_{\Omega} \theta_{0}(\mathbf{x}) d \mathbf{x}$, which is a constant. The proofs are in the spirit of this paper, we omit the details here.

REMARK 4.2. We note that Theorem 1.2 requires that $\beta(\phi)$ is a constant. It is interesting to study whether this condition can be removed or not. It seems a hard problem at the current stage. We leave the investigation for the future.

Acknowledgement. The author would like to thank the referees for their comments and suggestions on the improvement of the paper. K. Zhao was partially supported by NSF through grant DMS 0807406 and under Agreement No. 0635561.

\section{REFERENCES}

[1] N. Alikakos, P. Bates, and G. Fusco, Slow motion for the Cahn-Hilliard equation in one space dimension, J. Diff. Eqs., 90, 81-135, 1991.

[2] P. Bates and P. Fife, The dynamics of nucleation for the Cahn-Hilliard equation, SIAM J. Appl. Math., 53, 990-1008, 1993. 
[3] F. Boyer, Mathematical study of multi-phase flow under shear through order parameter formulation, Asymp. Anal., 20, 175-212, 1999.

[4] J. Cahn, On spinodal decomposition, Acta Metall., 9, 795-801, 1961.

[5] J. Cahn and J. Hilliard, Free energy of a nonuniform system. I. Interfacial free energy, J. Chem. Phys., 28, 258-267, 1958.

[6] J. Carr, M. Gurtin, and M. Slemrod, Structured phase transitions on a finite interval, Arch. Rational Mech. Anal., 86, 317-351, 1984.

[7] R. Chella and J. Vinals, Mixing of a two-phase fluid by a cavity flow, Phys. Rev. E, 53, 38323840, 1996.

[8] V. Cristini, H. Frieboes, J. Lowengrub, and S. Wise, Three-dimensional multispecies nonlinear tumor growth-I: Model and numerical method, J. Theo. Biol., 253, 524-543, 2008.

[9] V. Cristini, X.R. Li, J. Lowengrub, and S.M. Wise, Nonlinear simulations of solid tumor growth using a mixture model: Invasion and branching, J. Math. Biol., 58, 723-763, 2009.

[10] A. Debussche and L. Dettori, On the Cahn-Hilliard equation with a logarithmic free energy, Nonlin. Anal., 24, 1491-1514, 1995.

[11] C. Elliott and H. Garcke, On the Cahn-Hilliard equation with degenerate mobility, SIAM J. Math. Anal., 27, 404-423, 1996.

[12] X. Feng, Fully discrete finite element approximations of the Navier-Stokes-Cahn-Hilliard diffuse interface model for two-phase fluid flows, SIAM J. Numer. Anal., 44, 1049-1072, 2006.

[13] J. Goodman, H. Lee, and J. Lowengrub, Modeling pinchoff and reconnection in a Hele-Shaw cell. II. Analysis and simulation in the nonlinear regime, Physics of Fluids, 14, 514-545, 2002.

[14] J. Goodman, H. Lee, and J. Lowengrub, Modeling pinchoff and reconnection in a Hele-Shaw cell. I. The models and their calibration, Physics of Fluids, 14, 492-513, 2002.

[15] J. Gunton, M. San Miguel, and P. Sahni, Phase transitions and critical phenomena, in Domb, Lebowitz (Eds), Academic, London, 8, 1983.

[16] M. Gurtin, D. Polignone, and J. Vinals, Two-phase binary fluids and immiscible fluids described by an order parameter, Math. Mode. Meth. Appl. Sci., 6, 815-831, 1996.

[17] D. Kay and R. Welford, A multigrid finite element solver for the Cahn-Hilliard equation, SIAM J. Sci. Comput., 29, 288-304, 2007.

[18] K. Kang, J. Kim, and J. Lowengrub, Conservative multigrid methods for Cahn-Hilliard fluids, J. Comput. Phys., 193, 511-543, 2003.

[19] C. Liu and J. Shen, A phase field model for the mixture of two incompressible fluids and its approximation by a Fourier-spectral method, Physica D, 179, 211-228, 2003.

[20] J. Lowengrub and L. Truskinovsky, Cahn-Hilliard fluids and topological transitions, Proc. R. Soc. Lond. A, 454, 2617-2654, 1998.

[21] T. Ma and S.H. Wang, Cahn-Hilliard equations and phase transition dynamics for binary systems, Discrete Contin. Dyn. Syst. Ser. B, 11, 741-784, 2009.

[22] A. Miranville, A model of Cahn-Hilliard equation base on a microforce balance, C.R. Acad. Sci. Paris Sér. I Math., 328, 1247-1252, 1999.

[23] A. Onuki, Phase transitions of fluids in shear flow, J. Phys. Condens. Matter, 9, 6119-6157, 1997.

[24] J. Wei and M. Winter, Stationary solutions for the Cahn-Hilliard equation, Ann. Inst. Henri Poincaré, Anal. Non Linéaire, 15, 459-492, 1998.

[25] K. Zhao, Global regularity for a coupled Cahn-Hilliard-Boussinesq system on bounded domains, Quar. Appl. Math., 69, 331-356, 2010. 